\title{
Identificación de niveles de calidad de vida en personas con un rango de edad entre 50 y 85 años
}

Identification of levels of quality of life in people with a range of between 50 and 85

years old

Identificação dos níveis de qualidade de vida em pessoas com idade entre 50 e 85

anos

Jaqueline Guadalupe Guerrero Ceh

Universidad Autónoma de Campeche, México

jgguerre@uacam.mx

Ana Rosa Can Valle

Universidad Autónoma de Campeche, México

anarocan@uacam.mx

Betty Sarabia Alcocer

Universidad Autónoma de Campeche, México

bmsarabi@uacam.mx

\section{Resumen}

La calidad de vida es la percepción que cada persona tiene sobre su salud, emociones y, en general, bienestar. Es importante diferenciar la calidad de vida del nivel de vida, ya que el primero está relacionado con la percepción de cada sujeto y el segundo se refiere al aspecto económico. El objetivo aquí es identificar el nivel de calidad de vida que presentan las personas mayores. Para ello se aplicó en un solo momento la escala de calidad de vida WHOQOL-BREF a veintinueve personas mayores en un rango de edad de 50 a 85 años que acuden a dos grupos de apoyo diferentes. En los ítems No. 1 y 2, $21 \%$ (seis sujetos) obtuvieron valores menores a 3, calificando su calidad de vida como negativa y salud insatisfecha, en comparación con $79 \%$ que califica esos dos rubros (calidad de vida y salud) como normales y muy buenos. En el ítem No. 26, $21 \%$ de los sujetos de estudio muestra altos niveles de frecuencia de tristeza, sentimientos negativos y desesperanza, contrastando con el restante $79 \%$, que presenta satisfacción con su calidad de vida y salud, es decir, baja 
frecuencia de sentimientos negativos. Sólo 7 \% (dos personas) presenta una percepción negativa en los dos rubros. En conclusión, cerca del $80 \%$ de los sujetos de estudio se muestra satisfecho con su calidad de vida y percibe una salud acorde a su edad. Se identifica en riesgo al $7 \%$ de la población, al tener una autopercepción de su calidad de vida y salud como muy mala y muy insatisfecha. Se recomienda diseñar un programa de intervención gerontológica para evitar un riesgo mayor.

Palabras clave: Calidad de vida, salud, Personas Mayores.

\section{Abstract}

Quality of life is the perception that each person has your health, emotions and, in general, welfare. It is important to differentiate the quality of life of the standard of living, since the first is related to the perception of each subject and the second refers to the economic aspect. The objective is to identify the level of quality of life in older people. This was applied in a moment the quality of life, WHOQOL-BREF scale to twenty-nine elderly people in a range of 50 to 85 years old who attend two different support groups. In items no. 1 and 2, 21\% (six subjects) values obtained less than 3, as their quality of life as negative and unmet health, compared with $79 \%$ qualifying these two products (quality of life and health) as normal and very good. Item No. 26, 21\% of the subjects of study shows high levels of frequency of negative feelings, sadness and hopelessness, contrasting with the remaining $79 \%$, that shows satisfaction with their quality of life and health, i.e., low frequency of negative feelings. Only 7\% (two people) shows a negative perception in the two areas. In conclusion, about $80 \%$ of the subjects of study is satisfied with their quality of life and perceived health according to their age. Are identified at risk to $7 \%$ of the population, have a self-perception of their quality of life and health as a very bad and very dissatisfied. We recommend designing a gerontological intervention program to prevent one increased risk.

Key Words: quality of life, health, seniors.

\section{Resumo}

A qualidade de vida é a percepção de que cada pessoa tem sobre a sua saúde, emoções e bem-estar geral. É importante distinguir a qualidade de vida do nível de vida, uma vez que a primeira está relacionada com a percepção de cada sujeito e o segundo refere-se ao aspecto económico. O objetivo aqui é identificar o nível de qualidade de vida que apresentam os idosos. Para este foi aplicado em um único momento a escala de qualidade de vida WHOQOL-BREF a vinte idosos na faixa etária de 50-85 anos, alunos de dois grupos de apoio diferentes. Nos itens No. 1 e 2, 21\% (seis 
indivíduos) apresentaram valores inferiores 3, descrevendo a sua qualidade de vida como negativo e de saúde não atendidas, em comparação com $79 \%$ qualificando estes dois itens (qualidade de vida e saúde) como normal e muito bom. No item $n^{\circ} 26,21 \%$ dos sujeitos do estudo mostram uma alta frequência de tristeza, sentimentos negativos e desespero, em contraste com os restantes 79\%, que tem satisfação com a sua qualidade de vida e saúde, ou seja, de baixa frequência sentimentos negativos. Apenas 7\% (duas pessoas) tem uma percepção negativa nas duas áreas. Em conclusão, cerca de $80 \%$ dos sujeitos do estudo foram satisfeitos com a sua qualidade de vida e percebe uma saúde de acordo com a sua idade. Ele está em risco de $7 \%$ da população, tendo um auto-percepção de sua qualidade de vida e saúde como muito ruim e muito insatisfeito. Recomenda-se a projetar um programa de intervenção gerontológica para evitar mais riscos.

Palavras-chave: Qualidade de vida, saúde, Idosos.

Fecha recepción: Enero 2016

Fecha aceptación: Junio 2016

\section{Introducción}

Con frecuencia se relaciona la calidad de vida con la percepción económica. Es muy fácil confundir los términos calidad de vida y nivel de vida, pues ambos términos giran en torno a la persona; sin embargo, se aprecian diferencias sustanciales que seguidamente se explican: el nivel de vida está vinculado con el aspecto económico y la percepción monetaria del individuo, es decir, los aspectos externos, materiales y superficiales; por otro lado, el término calidad de vida se refiere a la percepción que tiene la persona sobre su condición de salud, un aspecto más relacionado con los sentimientos, lo interno.

La calidad de vida es: 
...la percepción individual de la propia posición en la vida dentro del contexto del sistema cultural y de valores en que se vive y en relación con sus objetivos, esperanzas, normas y preocupaciones. Es un concepto de amplio espectro, que incluye de forma compleja la salud física de la persona, su estado psicológico, su nivel de independencia, sus relaciones sociales, sus creencias personales y su relación con las características destacadas de su entorno (OMS, 1994, citado en OMS 2002, p. 98).

La calidad de vida está relacionada con lo que la persona percibe del mundo, de sus relaciones con las personas próximas y del trato que recibe por parte de todas las personas y las situaciones diversas en las que está inmersa.

A través del tiempo se ha intentado dar una definición que cubra todas las aristas del concepto calidad de vida, sus componentes subjetivos y objetivos donde el punto en común es el BIENESTAR individual. Los aspectos subjetivos se pueden agrupar en 5 dominios principales: el bienestar físico (como salud, seguridad física), bienestar material (privacidad, alimentos, vivienda, transporte, posesiones), bienestar social (relaciones interpersonales con la familia, las amistades, etcétera), desarrollo y actividad (educación, productividad, contribución) y bienestar emocional (autoestima, estado respecto a los demás, religión). Sin embargo, es importante comprender que la respuesta a cada uno de estos dominios es subjetiva y sumamente variable debido a la influencia de factores sociales y materiales, la edad misma de la persona, su situación de empleo o las políticas de salud (Instituto Nacional de Ciencias Médicas y Nutrición Salvador Zubirán, 2013, p.1).

Esta percepción que la persona tiene sobre su calidad de vida depende mucho de factores externos, como la economía y sus relaciones familiares, por mencionar algunos. Y va cambiando conforme se va perdiendo independencia financiera, sobre todo en las personas que gran parte de su vida han trabajado, sido productivas y totalmente independientes. ¿Qué sucede cuando la persona ya no tiene el mismo ritmo de vida, la misma rutina? ¿Qué pasa cuando la persona llega a la edad de su jubilación y ahora dispone de más tiempo pero de menos recursos? El panorama cambia considerablemente, ya que actualmente se percibe que la persona vale de acuerdo a la aportación económica que haga en la familia. Cuando disminuye su aportación disminuye su valor dentro de la familia; además están las patologías que van surgiendo por la edad y para las que requiere de 
recursos económicos para poder ser atendido. Entonces su situación se transforma y deja de aportar un "ingreso" para convertirse en un "egreso", una carga para la familia, en la cual si no está preparada psicológica y económicamente para afrontar esta nueva etapa, surgen más conflictos. Todo esto repercute en la salud de la persona mayor y en la forma como percibe su calidad de vida. Los expertos señalan que es importante prepararse anticipadamente a la jubilación, para disminuir el impacto negativo que se vive al jubilarse y para que la familia y la persona mayor perciba ese momento como una oportunidad de realizar actividades que no habían podido hacer por falta de tiempo.

El gerontólogo es el profesional del área de la salud que tiene las habilidades para realizar el trabajo previo que permita mejorar el escenario al momento de la jubilación, mediante la aplicación de estrategias en la familia y la persona mayor, y la creación de actividades y redes de apoyo. En este sentido, la familia es la primera y más importante red de apoyo, pues es la que ofrece la primera ayuda moral. Los familiares más cercanos pueden apoyar a la persona mayor a sentirse siempre acompañada y evitar que caiga en depresión. Si la familia falla o está desintegrada, entonces el Estado a través de diferentes instancias públicas debe colaborar en la atención de la persona mayor.

La siguiente tabla muestra un panorama general de los aspectos que definen el concepto de calidad de vida. 
Tabla 1. Diversas definiciones para el concepto de calidad de vida.

\begin{tabular}{|l|l|}
\hline \multicolumn{1}{|c|}{ REFERENCIA } & \multicolumn{1}{c|}{ DEFINICIÓN PROPUESTA } \\
\hline Ferrans (1990b) & $\begin{array}{l}\text { Calidad de vida general definida como el bienestar personal derivado de la satisfacción o } \\
\text { insatisfacción con áreas que son importantes para él o ella. }\end{array}$ \\
\hline Hornquist (1982) & $\begin{array}{l}\text { Define en términos de satisfacción de necesidades en las esferas física, psicológica, social, de } \\
\text { actividades, material y estructural. }\end{array}$ \\
\hline Shaw (1977) & $\begin{array}{l}\text { Define la calidad de vida de manera objetiva y cuantitativa, diseñando una ecuación que } \\
\text { determina la calidad de vida individual: QL=NE x (H+S), en donde NE representa la dotación } \\
\text { natural del paciente, H la contribución hecha por su hogar y su familia a la persona y S la } \\
\text { contribución hecha por la sociedad. Críticas: la persona no evalúa por sí misma, segundo, no } \\
\text { puede haber cero calidad de vida. }\end{array}$ \\
\hline Lawton (2001) & $\begin{array}{l}\text { Evaluación multidimensional, de acuerdo a criterios intrapersonales y socio-normativos, del } \\
\text { sistema personal y ambiental de un individuo. }\end{array}$ \\
\hline Haas (1999) & $\begin{array}{l}\text { Evaluación multidimensional de circunstancias individuales de vida en el contexto cultural y de } \\
\text { valor al que se pertenece. }\end{array}$ \\
\hline $\begin{array}{l}\text { Bigelow et al., } \\
\text { (1991) }\end{array}$ & $\begin{array}{l}\text { Ecuación en donde se balancean la satisfacción de necesidades y la evaluación subjetiva de } \\
\text { bienestar. }\end{array}$ \\
\hline Calman (1987) & $\begin{array}{l}\text { Satisfacción, alegría, realización y la habilidad de afrontar... medición de la diferencia, en un } \\
\text { tiempo, entre la esperanza y expectativas de una persona con su experiencia individual } \\
\text { presente. }\end{array}$ \\
\hline $\begin{array}{l}\text { Martin \& Stockler } \\
\text { (1998) }\end{array}$ & $\begin{array}{l}\text { Tamaño de la brecha entre las expectativas individuales y la realidad a menor intervalo, mejor } \\
\text { calidad de vida. }\end{array}$ \\
\hline Opong et al., (1987) & Condiciones de vida o experiencia de vida. \\
\hline
\end{tabular}

Tomado de Urzúa, A. y Caqueo-Urizar, A. (2012). Calidad de vida, una revisión teórica al concepto. http://www.scielo.cl/pdf/terpsicol/v30n1/art06.pdf

\section{OBJETIVO}

Identificar el nivel de calidad de vida que presentan las personas mayores.

\section{MATERIALES Y MÉTODOS}

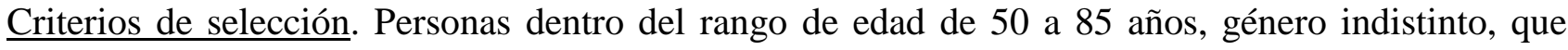
acudan a los grupos de apoyo ubicados en los siguientes lugares: Centro de Desarrollo Comunitario de Leovigildo Gómez y Asociación de Jubilados y Pensionados del ISSSTE que se reúnen en las instalaciones de la CNOP, en la Ciudad de San Francisco de Campeche, Campeche, México.

Instrumento. Se utilizó el Instrumento de medición de calidad de vida Whoqol-Bref, el cual está integrado por tres apartados: la primera parte son los datos de identificación como género, fecha de nacimiento, estudios, estado civil, problemas de salud, aspecto laboral; la segunda parte inicia con las indicaciones para resolver las cinco categorías o dimensiones: I. Integra dos ítems: No. 1 y 2 , relacionada con su calidad de vida y satisfacción con su salud; II. Integra siete ítems: No. 3, 4, 5, 6, 7, 8 y 9 relacionados con su percepción del dolor, disfrute de la vida, concentración y ambiente 
físico, entre otros aspectos; III. Integra seis ítems: No. 10, 11, 12, 13, 14 y 15, relacionado con independencia física; IV. Integra diez ítems: No. 16, 17, 18, 19, 20, 21, 22, 23, 24 y 25, relacionado con la satisfacción a través de las actividades de la vida diaria (AVD), vida sexual, apoyo de amig@s condiciones del lugar donde vive, entre otros aspectos; V. Integrado por un ítem: No. 26, relacionado con la frecuencia de sentimientos negativos; un total de 26 ítems de opción múltiple. Se pide a la persona que resuelva pensando en las dos últimas semanas en su modo de vivir, expectativas, placeres y preocupaciones; las categorías que integran esta segunda parte se dividen en dominios: físico, psicológico, relaciones sociales y entorno. Todos los ítems tienen cinco opciones de respuesta, a continuación se dan a conocer las opciones:

Muy mala, Mala, Normal, Buena y Muy Buena

Muy satisfecho, Insatisfecho, Ni satisfecho ni insatisfecho, Satisfecho, Muy satisfecho

Nada, Un poco, Moderado, Bastante, Totalmente

La tercera parte está integrada por tres preguntas abiertas, las cuales permiten identificar los aspectos siguientes: si recibió ayuda, el tiempo en responder el instrumento, finalizando con un espacio para que la persona que responde, escriba sus comentarios sobre el cuestionario.

La escala de calidad de vida Whoqol-Bref es versión original de la Organización Mundial de la Salud, la versión española es de Lucas R. en 1998, y ha sido utilizada en investigaciones diversas. Es útil en la valoración de la vida en general y de la satisfacción en salud de la persona que lo responde. Al final de la aplicación se suman todos los valores y se interpreta que a mayor puntuación, la calidad de vida es mejor.

Para el análisis de los resultados se consideraron cuatro dimensiones, las número: I, III, IV y V. Para la dimensión I, se tomó como criterio la suma de los dos ítems, considerando un mínimo de 6 puntos como aceptable, dado que los valores de 3 en cada rubro corresponde a Normal y ni satisfecho ni insatisfecho. Para la dimensión III, se observa que a más valor es más positiva su percepción, tomando en consideración que son seis ítems y que la opción mínima aceptada es "moderado" con un valor de 3 puntos cada uno; se considera como mínimo aceptado 20 puntos en total en esta dimensión. Para la dimensión IV, integrados por diez ítems con un valor de 3 puntos 
cada uno, se considera como mínimo aceptable 35 puntos. Para la dimensión $\mathrm{V}$, integrada por un ítem, se considera como aceptable un valor máximo de 3 puntos, valores menores a tres es bueno.

\section{Resultados}

Figura 1. Género de la población.

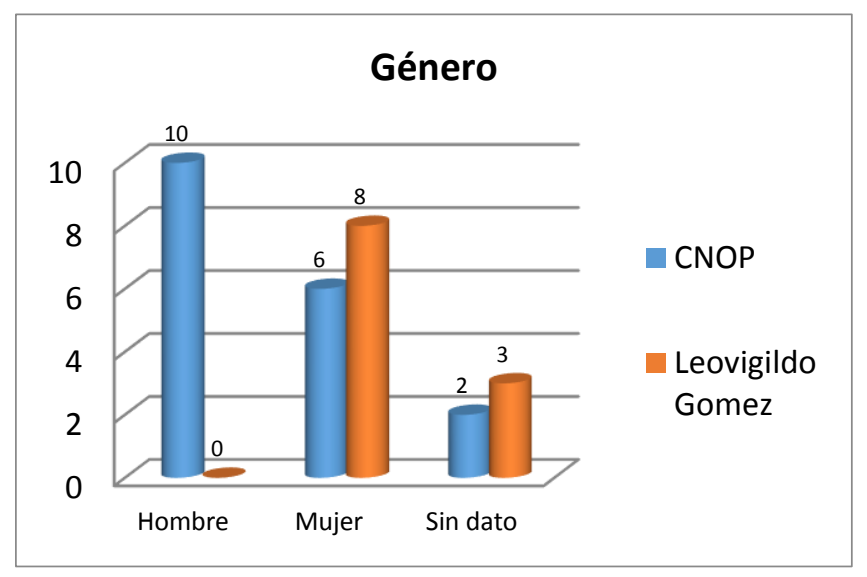

En la figura No. 1 se observa que de los veintinueve sujetos de estudio, $17 \%$ (5) no puso el dato de género, de los veinticuatro restantes $35 \%$ (diez) es del género masculino y $48 \%$ (14) del género femenino. Hubo una ligera prevalencia del género femenino.

Figura 2. Nivel de estudios de la población.

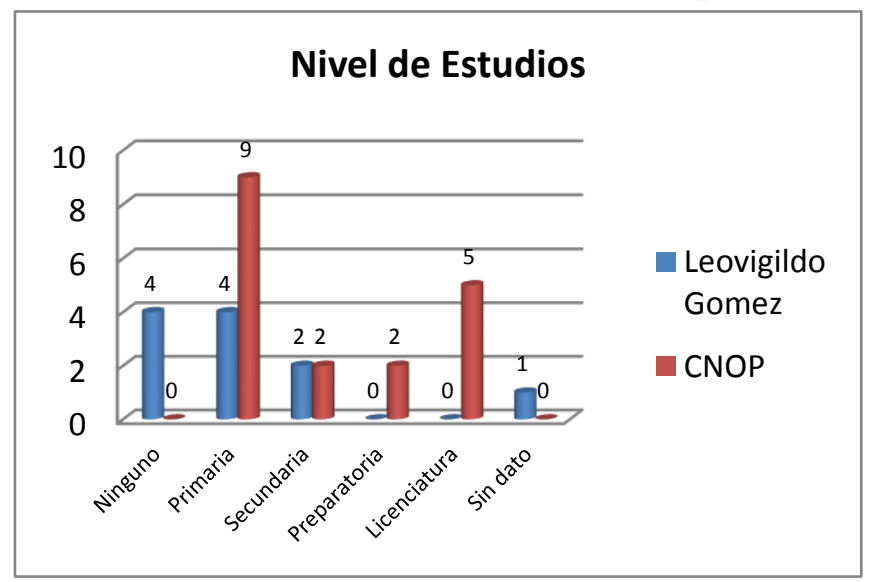


En la figura No. 2 se observa que de los veintinueve sujetos de estudio, $3 \%$ (1) está sin dato, reduciéndose a 28 las personas que proporcionaron información, de las cuales: 14 \% (4) se refiere sin estudios, $45 \%$ (13) tiene estudios de nivel primaria, $14 \%$ (4) de secundaria, $7 \%$ (2) de nivel medio superior y $17 \%$ (5) de nivel superior. Se observa que las personas respondieron como nivel superior el hecho de haber estudiado la Normal de Profesores, dado que en su mayoría en la Sede de la CNOP son del ámbito magisterial, una de las pocas opciones de estudio a su alcance.

Figura 3. Estado civil de la población.

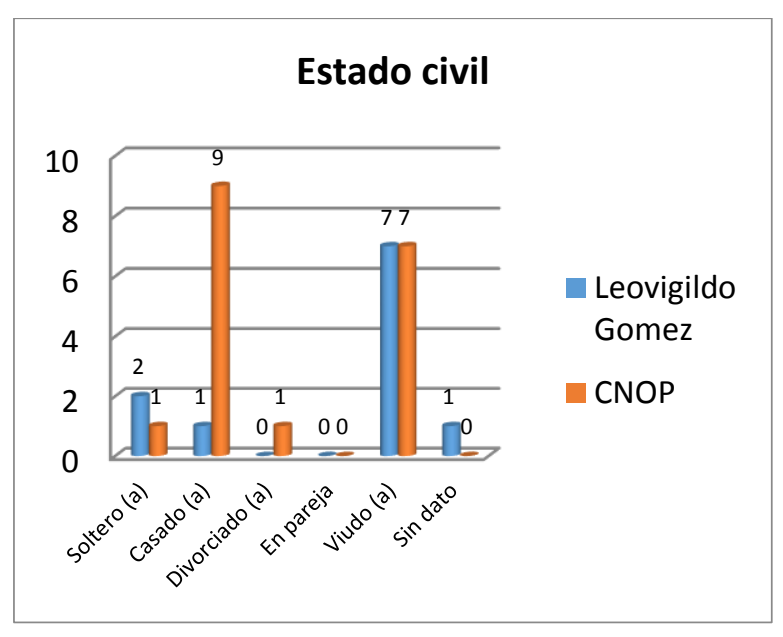

La figura No. 3 muestra el estado civil de los sujetos de estudio, de los cuales $3 \%$ (1) no proporcionó dato, $10 \%$ (3) está solter@, 34 \% (10) casad@, 3\% (1) divorciad@, 48\% (14)viud@. Un total de 18 personas se refirieron sin pareja, es decir, $62 \%$ de la población.

Figura 4. Autopercepción de enfermedad.

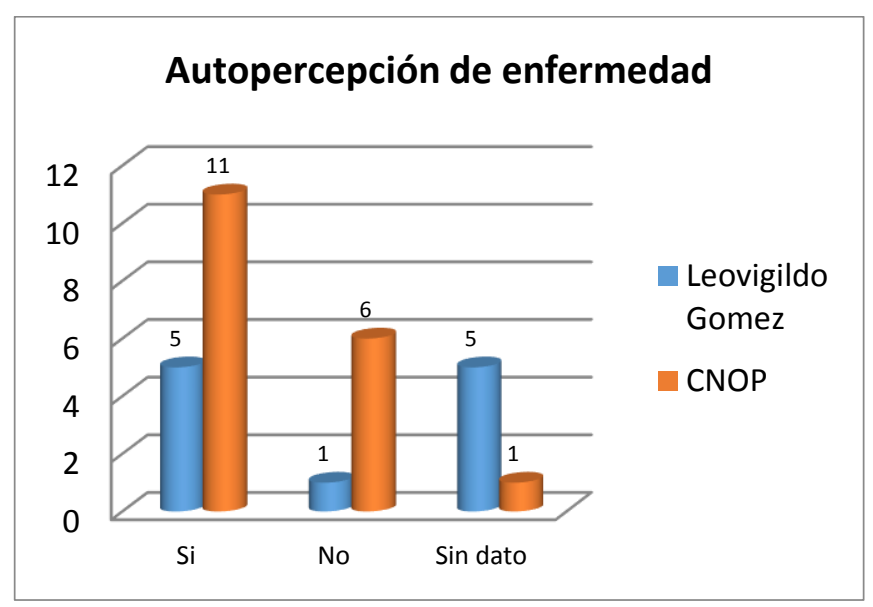


La figura No. 4 también corresponde a la parte previa de la escala de calidad de vida y se refiere a responder si actualmente está enferm@. Por una parte, $55 \%$ (16) respondió afirmativamente y $24 \%$ (7) negativamente, y el restante $21 \%$ (6) no dio información.

Figura 5. Resultados por dimensión en la sede Leovigildo Gómez

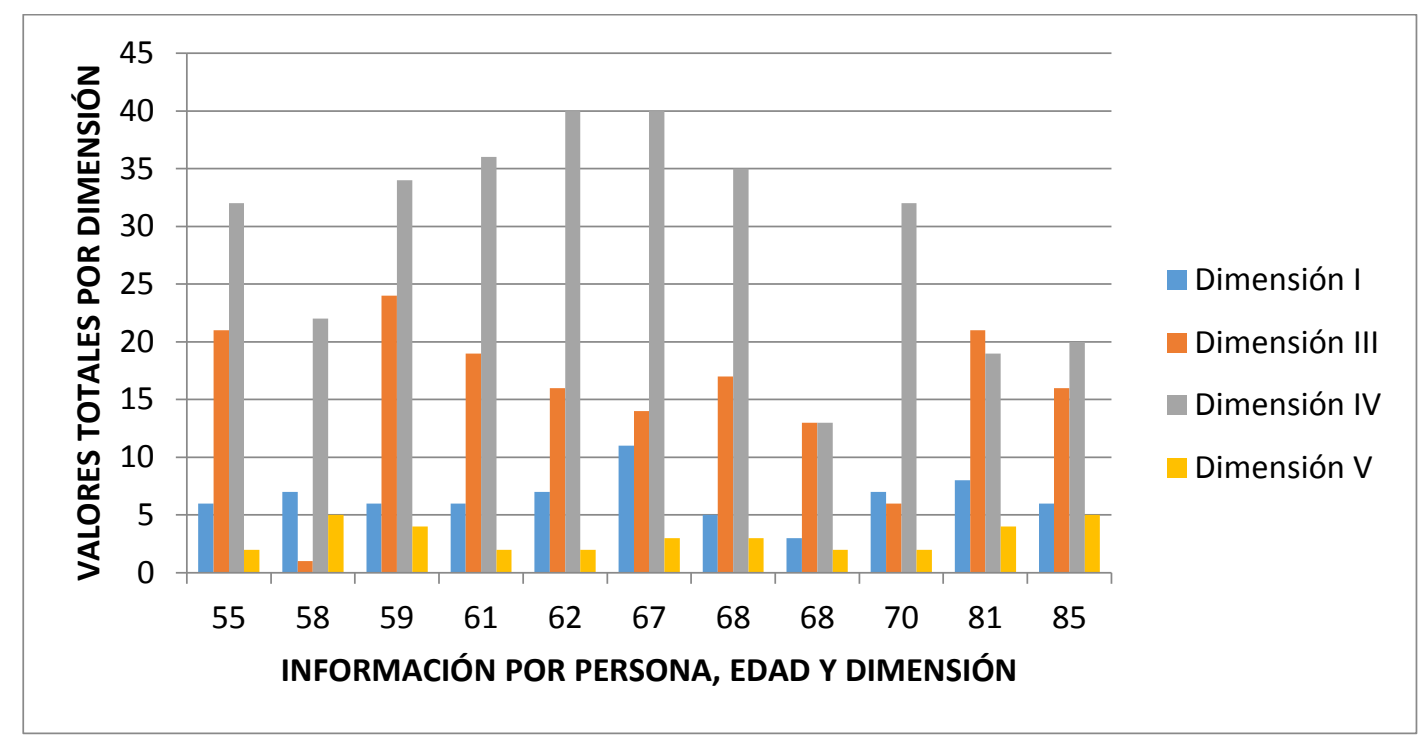

La figura No. 5 muestra los resultados de los once sujetos de estudio que asisten a la sede en la colonia Leovigildo Gómez, de acuerdo a su edad y dimensión analizada.

Figura 6. Resultados por dimensión en la sede CNOP.

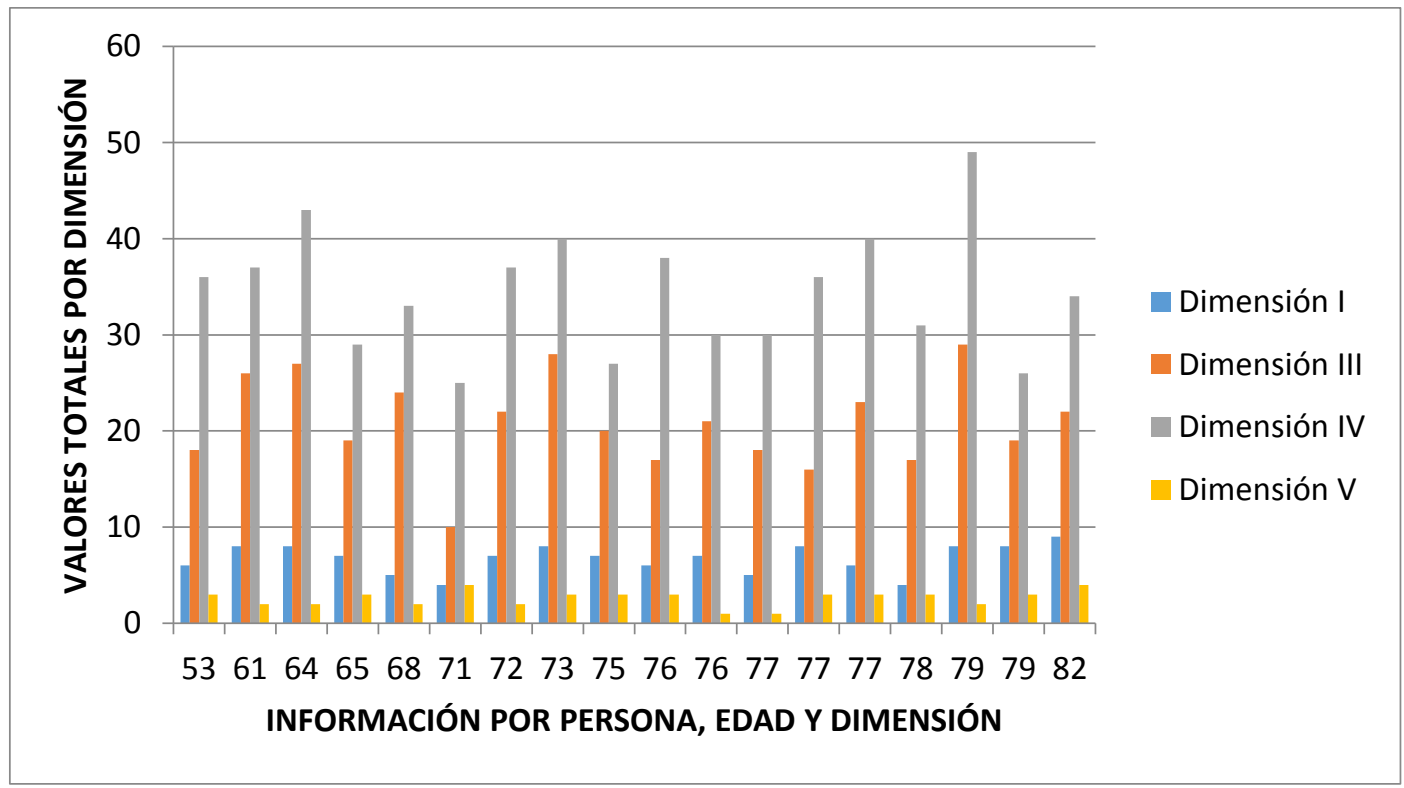


La figura No. 6 muestra los resultados de los dieciocho sujetos de estudio que asisten a la sede de la CNOP, de acuerdo a su edad y dimensión analizada.

Análisis por dimensiones

Dimensión I, integrada por dos ítems No. 1 y 2, $21 \%$ (seis sujetos: 4 de CNOP + 2 de Leovigildo) obtuvieron valores menores a 3 , calificando su calidad de vida como negativa y salud insatisfecha, en comparación con 79 \% (veintitrés sujetos: 14 de CNOP + 9 de Leovigildo) que calificó esos dos rubros (calidad de vida y salud) como normal y muy bueno. Se toma como mínimo el valor de seis puntos para considerarlo como sin riesgo.

Dimensión III, integrada por seis ítems del 10 al 15, para considerar sin riesgo, se ha considerado el valor mínimo de veinte puntos. Por otro lado, $55 \%$ (dieciséis sujetos: 8 de CNOP +8 de Leovigildo) obtuvo valores máximos de diecinueve, no logrando alcanzar la mínima de veinte. Asimismo, $45 \%$ (trece personas: 10 de CNOP + 3 de Leovigildo) obtuvo valores en un rango de veinte a veintinueve puntos.

Dimensión IV, integrada por diez ítems del 16 al 25, para considerar sin riesgo, se ha considerado el valor mínimo de treinta y cinco puntos. Más de la mitad, $55 \%$ (dieciséis sujetos: 8 de CNOP +8 de Leovigildo) obtuvo valores máximos de treinta y cuatro, no logrando alcanzar la mínima de treinta y cinco. Además, $45 \%$ (trece personas: 10 de CNOP + 3 de Leovigildo) obtuvo valores en un rango de treinta y cinco a cuarenta y nueve puntos.

Dimensión VI, integrada por un ítem, el No. 26, para considerar sin riesgo se ha considerado el valor máximo tres puntos. Así, 79 \% (veintitrés sujetos: 16 de CNOP + 7 de Leovigildo) obtuvo valores máximos de tres; $21 \%$ (seis personas: 2 de CNOP + 4 de Leovigildo) alcanzó valores en un rango de cuatro a cinco puntos.

\section{Conclusiones}

Cerca del $80 \%$ de los sujetos de estudio se muestra satisfecho con su calidad de vida y percibe una salud acorde a su edad. Sin embargo, se identifica en riesgo al $7 \%$ de la población, ya que expresar una autopercepción de su calidad de vida y salud como muy mala y muy insatisfecha. Se recomienda diseñar un programa de intervención gerontológica para evitar un riesgo mayor. Las dimensiones III y IV coinciden en valores porcentuales. En la sede Leovigildo se observa que sólo $27 \%$ (3 personas) obtuvo valores arriba de 35 que es el mínimo, lo cual refleja que se debe trabajar 
con esta sede en los aspectos salud y autoestima. Es importante que se apliquen otras escalas que permitan identificar las áreas de oportunidad de estas personas mayores, con la intención de mejorar su autopercepción de la calidad de vida.

Finalmente, se recomienda a los investigadores evitar aplicar directamente las escalas, debido a que se corre el riesgo de obtener una información sesgada. Las personas mayores necesitan ver la utilidad de lo que hacen y el tiempo que le dedican, por esta razón se sugiere tener un acercamiento con ellos a través de una charla informal que les sea útil y los sensibilice, y así garantizar un mejor resultado.

\section{Agradecimientos}

Fue posible analizar estos datos gracias a la participación de los estudiantes de la licenciatura en gerontología: Alexandra, Tania, Aria, Fernando, Gladys, Habib, Lupita, y el Gerontólogo Daniel Muñoz González, quienes estuvieron en contacto con las personas mayores en ambas sedes de estudio. 


\section{Bibliografía}

Cardona, D. y Byron, H. (2005). Construcción cultural del concepto calidad de vida. Revista Facultad Nacional de Salud Pública, vol. 23, no. 1. Consulta realizada el 25 de junio de 2016. Disponible en http://www.scielo.org.co/pdf/rfnsp/v23n1/v23n1a08.pdf

Gildenberger, C. (1978). Desarrollo y Calidad de Vida. En: Revista Argentina de Relaciones Internacionales, No. 12. CEINAR. Buenos Aires, Argentina. Consulta realizada el 10 de junio de 2016. Disponible en http://www.cricyt.edu.ar/enciclopedia/terminos/CaliVida.htm

Herrera, A. P., y Guzmán, A. H. (2012). Reflexiones sobre calidad de vida, dignidad y envejecimiento. (Spanish). Revista Médica Clínica Las Condes, 23(1), 65-76.

Hernández, Zoila (2012). Estrés en Personas Mayores y Estudiantes Universitarios: un Estudio Comparativo. Consulta realizada el 17 de mayo 2016. Disponible en: http://www.redalyc.org/pdf/1339/133915936007.pdf

Instituto Nacional de Ciencias Médicas y Nutrición Salvador Zubirán (2013). ¿Qué es calidad de vida?, p. 1. Consulta realizada el 08 de junio de 2016. Disponible en http://www.innsz.mx/opencms/contenido/investigacion/comiteEtica/calidadVida.html

Luciano Magalhães Vitorino; Lisiane Manganelli Girardi Paskulin; Lucila Amaral Carneiro Vianna Artigo (2012). Calidad de vida de mayores en institución de larga permanencia. Revista Latino-Americana Enfermagem (RLAE) V20N6:1186-1195 - Artículos Originales. Consulta realizada el 2 de junio de 2016. Disponible en http://rlae.eerp.usp.br/index/search

Magollon, E. (2012). Una perspectiva integral del adulto mayor en el contexto de la educación. documenting electronic sources on the internet. Consulta realizada el 17 de mayo 2016. Disponible en: http://www.crefal.edu.mx/rieda/images/rieda-2012-1/contrapunto1.pdf 
Organización Mundial de la Salud (2002). Programa Envejecimiento y Ciclo Vital. Envejecimiento activo: un marco político. Revista Especializada en Geriatría y Gerontología no. 37(S2):74105. Consulta realizada el 15 de junio de 2016. Disponible en

http://www.imserso.es/InterPresent2/groups/imserso/documents/binario/docintenvejecimiento.pdf

Urzúa, A. y Caqueo-Urízar, A. (2012). Calidad de vida, una revisión teórica al concepto. Revista Terapia Psicológica 2012, vol. 30, no. 1, pp. 61 - 71. Sociedad Chilena de Psicología Clínica. Consulta realizada el 22 de junio de 2016. Disponible en http://www.scielo.cl/pdf/terpsicol/v30n1/art06.pdf

Zea, C., López-Alzate, M., Marín, C., Gómez, J., Aguirre-Acevedo, D., Restrepo, F. y Rupprecht, R. (2008). Autovaloración de calidad de vida y envejecimiento en adultos con riesgo de Alzheimer/Self-assessment of quality of life and aging of Alzheimer development risk adults. (Investigacion / Research). Investigación y Educación en Enfermería, 26(1), 24+. Retrieved from

http://go.galegroup.com/ps/i.do?id=GALE\%7CA200117759\&sid=summon\&v=2.1\&u=pu\&it=r\&p= AONE\&sw=w\&asid=86c3214008117cc25046df90ecf659f0 